\title{
Discovery and mechanism of action of pegvisomant
}

John J Kopchick

Edison Biotechnology Institute and Department of Biomedical Sciences, College of Osteopathic Medicine, Ohio University, Athens, Ohio 45701, USA

(Correspondence should be addressed to J J Kopchick; Email: kopchick@ohio.edu)

\begin{abstract}
Using a structure-function approach to the understanding of the molecular topology of the GH molecule, we discovered that glycine in the third $\alpha$-helix of GH (G119 of bovine GH and G120 of human $\mathrm{GH}$ ) was an important amino acid required for GH action. Substitution of this glycine residue with a variety of amino acids results in molecules that lack growth-promoting activity. More importantly, these molecules inhibit the actions of GH both in vitro and in vivo. These results, obtained more than a decade ago, were the basis for the discovery of GH antagonists. Since that time, efforts have been focused on establishing the mechanism by which these antagonists inhibit GH action. In this regard, in vivo expression of GH-antagonist genes in transgenic mice results in dwarf animals. The animals are fertile and possess no abnormal 'phenotypes'. Dwarf mice have also been created by disrupting or 'knocking out' the GH receptor gene. Together, these results have laid the foundation for the clinical use of GH antagonists when endogenous GH levels are increased or when GH is known to be a factor in the progression of the disorder.
\end{abstract}

European Journal of Endocrinology 148 S21-S25

\section{Introduction}

The journey of scientific discovery that led to the development of pegvisomant, a growth hormone (GH) receptor (GHR) antagonist, began approximately 15 years ago with a series of experiments aimed at elucidating the structure-function relationship of GH. This $22 \mathrm{kDa}$ protein is secreted from the pituitary gland in an episodic pattern and is central to the regulation of growth (Fig. 1) (1, 2). It also has numerous other biological effects, including enhancement of milk production, nitrogen retention, lipolysis and diabetogeniclike effects (3). GH exerts its activity either directly on tissues such as liver, muscle, bone or fat to induce metabolic changes, or indirectly through the induction of insulin-like growth factor-I (IGF-I) expression, which also is a growth-promoting factor (Fig. 1) (1). This review outlines the experimental strategy that led to the discovery of pegvisomant, the first GHR antagonist to be used clinically.

\section{Structure of GH and GHR}

The 3-dimensional structure of $\mathrm{GH}$ was first determined in 1987 by X-ray crystallography, employing a genetically engineered variant of porcine (p) GH (4). The 191 amino acid pGH protein consists of four $\alpha$-helices, ranging from 20 to 30 residues each, joined together by stretches of nonhelical polypeptide chains, which are tightly packed together in an antiparallel bundle
(Fig. 2) (4). There are two disulfide bridges in the protein, the first between Cys residues 53 and 164, the second between Cys residues 181 and 189 (4).

GHR is a transmembrane protein with an extracellular domain that binds to $\mathrm{GH}$; the intracellular domain interacts with other proteins in a signal transduction cascade that controls cellular functions of the target cell (5). The binding relationship between $\mathrm{GH}$ and GHR was determined by X-ray crystallography, using the extracellular domain of the GHR bound to GH. In the crystals of the human (h) GH-receptor complex, a single hGH molecule is bound to the GHR in a ratio of $1: 2$, suggesting dimerisation of the receptor $(6,7)$. Further analysis shows that GH has two binding sites located on opposite sides of the bundle of $\alpha$-helices (8). Binding of hGH to the two GHRs is thought to be sequential; site 1 on hGH binds one GHR first, followed by site 2 binding the second $\operatorname{GHR}(7,8)$.

\section{Structure-function relationship of $\mathbf{G H}$}

Mutagenic studies were conducted to determine the relationship between the structure and function of $\mathrm{GH}$ in parallel with the studies aimed at determining the 3-dimensional structure of GH. Identification of the appropriate amino acids within the sequence to modify is one of the difficulties encountered in this type of study. Considerable homology exists between $\mathrm{GH}$ amino acid sequences from different species, particularly in the $\alpha$-helical regions. This suggests 


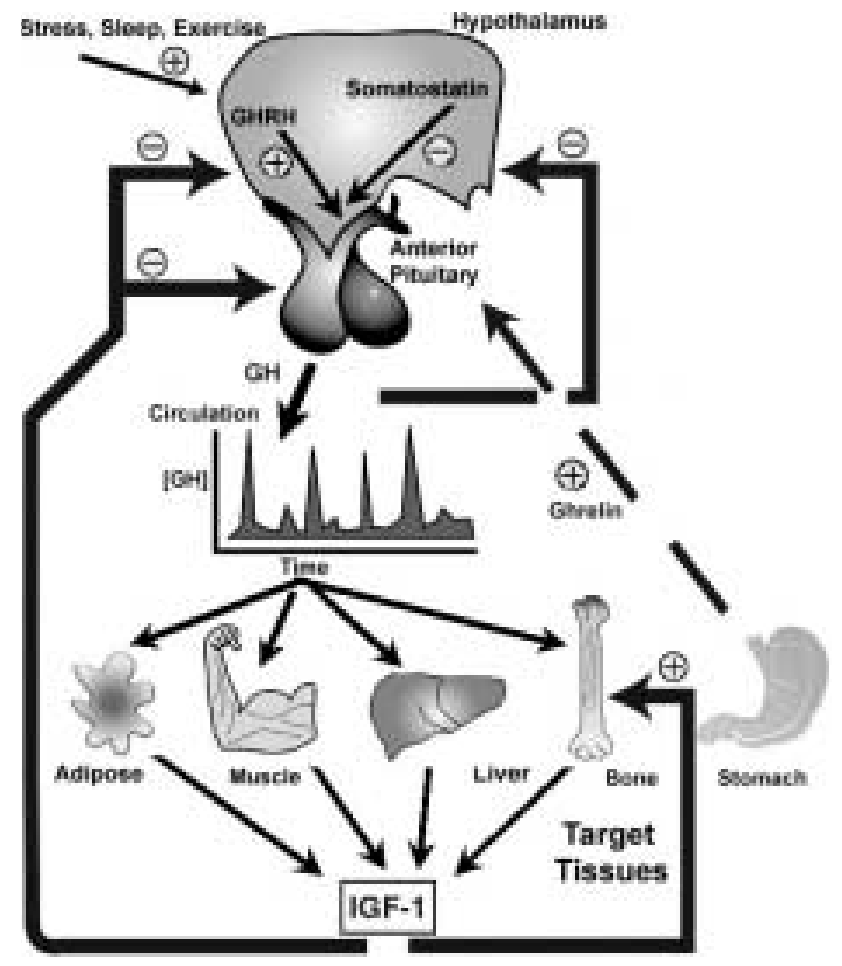

Figure 1 Schematic depiction of the regulation of $\mathrm{GH}$ secretion and effects of $\mathrm{GH}$ within the body. $\mathrm{GH}$ is released in a pulsatile manner and then binds to GHRs on target tissue, including adipose, muscle, liver and bone. GH also promotes secretion of IGF-I that also stimulates growth processes. IGF-I inhibits further $\mathrm{GH}$ release via a long negative-feedback loop. (Reprinted, with permission, from (2).)

that these regions are important for the structural and functional integrity of GH (4). Alignment of the third $\alpha$-helix into a 2-dimensional Edmundson wheel (axial) projection reveals an amphipathic segment of amino acids between residues 109 and 126 of bovine (b) GH (bGH (109-126)). An amphipathic segment of protein has hydrophilic amino acids clustered together and hydrophobic residues clustered separately in the axial projection of the helix. In bGH (109-126), however, amino acid residues 117,119 and 122 are positioned so that an imperfect amphipathic $\alpha$-helix is produced (Fig. 3A). Changing an imperfect amphipathic $\alpha$-helix to a perfect $\alpha$-helix had previously been shown to increase the potency of certain molecules such as GH-releasing factor (9). A series of studies was thus undertaken to determine whether modification of these three residues in the GH molecule that generate a perfect amphipathic $\alpha$-helix would alter the growth-related properties of bGH. A mutated bGH gene was engineered in which the codon for Glu-117 was replaced with Leu (E117L), Gly-119 was replaced with Arg (G119R), and Ala-122 was replaced with Asp (A122D) ( $\left.3^{\Delta} \mathrm{bGH}\right)(10)$. The resulting molecule possessed a perfect amphipathic $\alpha$-helix with all

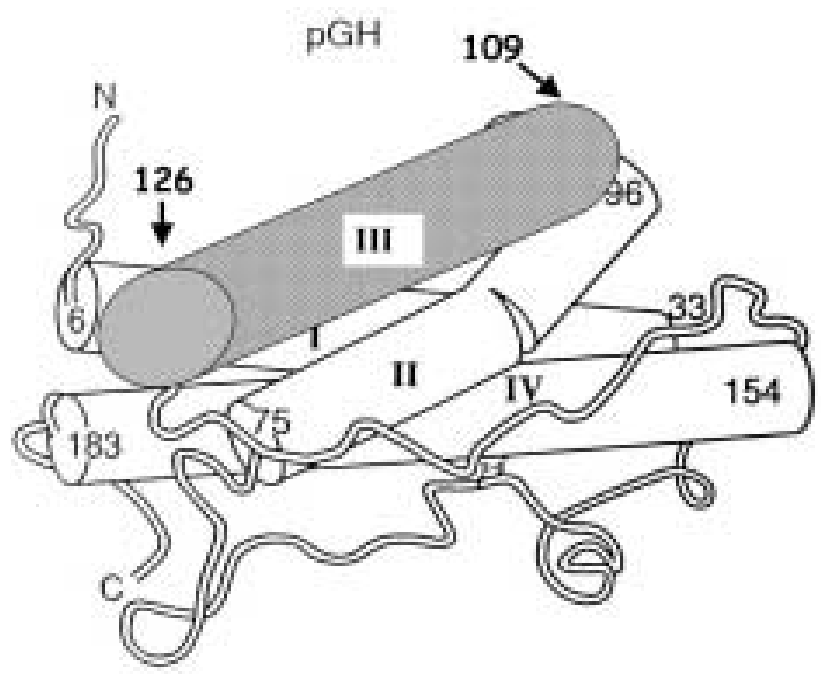

Figure 2 Schematic representation of the structure of $\mathrm{GH}$. The four $\alpha$-helices are drawn as cylindrical rods, while the regions of nonhelical polypeptide are shown as thin tape. The third $\alpha$-helix is formed by residues 109-126. (Reprinted, with permission, from (2).)

hydrophobic residues below and hydrophilic residues above the midline of the helix (Fig. 3B).

The $3^{\Delta} \mathrm{bGH}$ analogue was then characterized in vitro and in vivo. Data from in vitro GHR binding studies showed no significant difference between wild-type bGH and $3^{\Delta} \mathrm{bGH}$ in their GHR interactions, indicating that $3^{\Delta}$ bGH had a binding affinity similar to wild-type GH. Surprisingly, however, when the gene encoding $3^{\Delta} \mathrm{bGH}$ analogue was expressed in transgenic mice, it resulted in growth retardation; the transgenic mice that expressed the mutated GH were significantly smaller compared with nontransgenic littermates (10). This result was contrary to the expectation that the $3^{\Delta} \mathrm{bGH}$ transgenic mice would be larger than normal. When pituitary levels of mouse (m) GH were determined in these transgenic mice, GH levels were relatively high but serum IGF-I levels were low (11). The expression of $\mathrm{mGH}$ in the pituitary gland is controlled by IGF-I as part of a classic negative-feedback system. When GH is released, it interacts with the GHR, stimulating production of IGF-I in the target cells. If GH interaction with its receptor is inhibited and IGF-I is not expressed, then the negative feedback on GH production in the pituitary gland does not occur, resulting in increased $\mathrm{GH}$ concentrations. Thus, in the $3^{\Delta} \mathrm{bGH}$ transgenic mice, $3^{\Delta}$ bGH was acting as an in vivo antagonist to $\mathrm{mGH}$ activity, the first such functional GH antagonist to be identified.

The explanation as to why the mutated GH was unable to elicit a growth response was unknown. It bound the receptor with a high affinity and with similar kinetics to the wild-type $\mathrm{GH}$ in vitro, but the three amino acid substitutions in GH appeared to prevent 

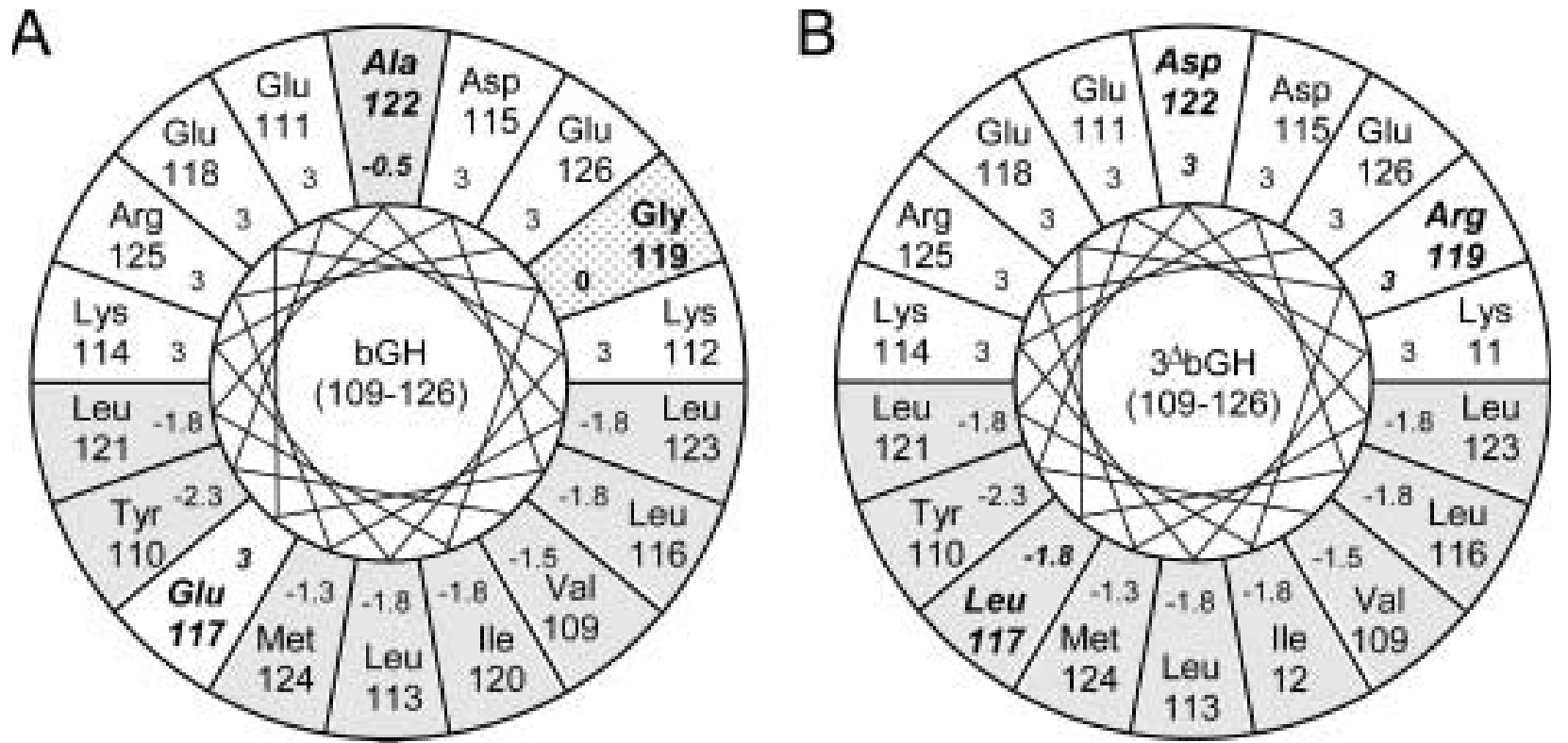

Figure 3 Edmundson wheel projection of the third $\alpha$-helix of $(A)$ wild-type bGH, and (B) the mutated $G H\left(3^{\Delta}\right.$ bGH). Amino acids are depicted with their position in the polypeptide chain and the corresponding hydrophilicity values. Shaded residues are hydrophobic. The Gly residue is nonpolar and is depicted with dots. (Reprinted, with permission, from (10).)

activation of the receptor (10). Structural studies then demonstrated that dual binding sites on hGH to two GHRs are required for receptor dimerisation and activation, which results in growth-promoting activities $(7,8)$. Thus, one GH molecule interacting with two GHRs are required for subsequent intracellular signalling that ultimately results in the various phenotypes associated with GH action. One should note that the GH antagonist does not inhibit GHR dimerisation but prevents proper dimerisation $(12,13)$.

Although the structure of $3^{\Delta}$ bGH had been characterised, it was not clear which one or more of the three amino acid changes was required for the antagonistic activity. Unique GH analogues were generated for each of the amino acid residues in question. As with the triple-mutation analogue, all three of the single mutated analogues bound to the GHR with similar affinities. However, it was only when the G119 residue was substituted (bGH-G119R) that a dwarf transgenic mouse was produced (14). Thus functional antagonism of the GHR in the $3^{\Delta} \mathrm{bGH}$ analogue was determined by the amino acid in the G119R mutation. Similar studies were performed with hGH where altering hGH Gly-120 to Arg resulted in an hGH antagonist (15).

\section{GH as an antagonist: the role of Gly-119 substitution}

Gly-119, which corresponds to Gly-120 in hGH, is invariant in the amino acid sequences of $\mathrm{GH}$ family members (16), suggesting that this residue is crucial for the functioning of GH. Gly is the smallest amino acid and lacks a bulky side chain. In a space-filling model of hGH, Gly-120 is positioned alongside an Ala residue (the second-smallest amino acid), producing a substantial cleft in the 3-dimensional model of the protein (Fig. 4). Mutational studies showed that replacing this Gly with several amino acids other than Ala resulted in an hGH antagonist (14). A subsequent report confirmed that replacing hGH G120 with arginine resulted in a GH antagonist (17). In the co-crystal

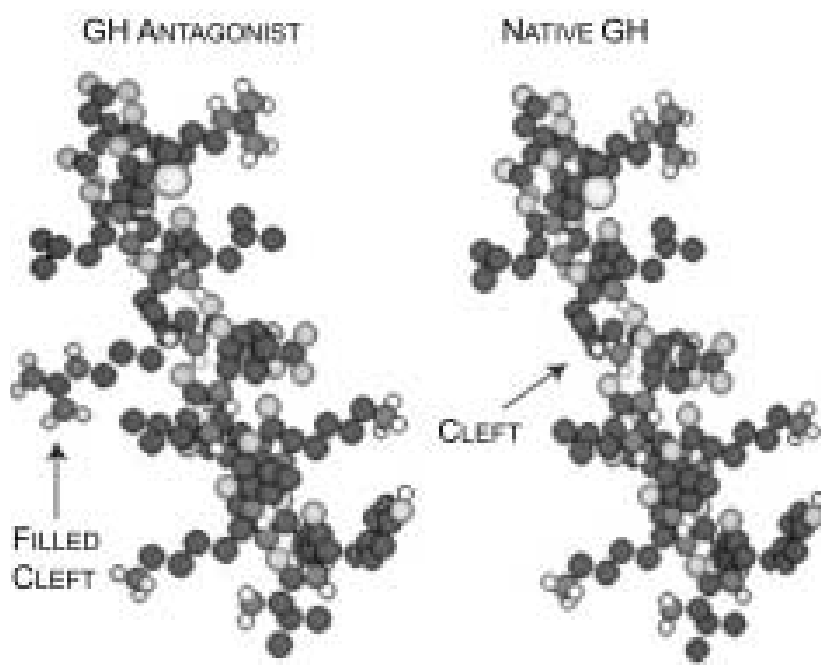

Figure 4 Space-filling models of GH and the mutated GH antagonist, showing the cleft that occurs naturally in the third $\alpha$-helix of $\mathrm{GH}$ because of the presence of Gly, and the same cleft filled in the $\mathrm{GH}$ antagonist by an amino acid with a large side group. (Reprinted, with permission, from (14).) 
structure of the GH-receptor complex, the cleft in the third $\alpha$-helix interacts with a Trp residue in the GHR molecule (Trp-104). Any residue larger than an Ala or Gly residue at residue 120 of hGH results in disruption of the proper binding of the second receptor; thus no functional receptor dimerisation or subsequent intracellular signalling $(12,13)$.

\section{The first GHR antagonist: pegvisomant}

Like wild-type hGH, genetically engineered variants of GH are rapidly cleared by the kidney and have very short elimination half-lives. This potentially limits their usefulness as GH antagonists. To overcome these problems, the GH antagonist (hGH G120K) was pegylated, that is, four or five polyethylene glycol (PEG) residues were added to the molecule. These PEG molecules are covalently attached to Lys residues producing a molecule with a mass of $42-46 \mathrm{kDa}(18,19)$.

In addition to the single amino acid modification of binding site 2 (G120R) discussed earlier, the pegvisomant molecule has modifications at binding site 1 that increase binding affinity and give it a competitive binding advantage over wild-type hGH (reviewed by Wells and colleagues (3)). The eight substitutions in binding site 1 are H18D, H21N, R167N, K168A, D171S, K172R, E174S, I179T (20, 21). It has recently been suggested that the crucial changes are K168A and K172R, both Lys residues. Substitution of these Lys residues with other amino acid removed potential PEG addition sites (13). The resulting GHR antagonist has been termed pegvisomant.

An additional concern is that the introduction of a modified GH protein into patients could trigger the formation of antibodies. In long-term clinical studies, antibodies were detected in 27 of 160 patients treated with pegvisomant (16.9\%); however, tachyphylaxis was not seen (23).

The results of the above-described studies laid the foundation for the clinical use of GHR antagonists in acromegaly. Pegvisomant, the first GHR antagonist available for clinical development, has a prolonged half-life, does not induce antibodies to itself, and acts as an antagonist at the GHR in vitro and in vivo $(1,19,21)$. Pegvisomant has been shown to effectively reduce serum IGF-I concentrations in both short- and long-term clinical studies, demonstrating its efficacy in greater than $97 \%$ of patients in the treatment of acromegaly $(22,23)$. A recent review concerning the discovery and use of $\mathrm{GH}$ antagonists has been presented (24).

\section{Acknowledgements}

JJK is supported, in part, by the State of Ohio's Eminent Scholar programme, which includes a gift by Milton and Lawrence Goll and by the Sensus Corp.

\section{References}

1 Okada S \& Kopchick JJ. Biological effects of growth hormone and its antagonist. Trends in Molecular Medicine 20017 126-132.

2 Kopchick JJ \& Andry JM. Growth hormone (GH), GH receptor, and signal transduction. Molecular Genetics and Metabolism 200071 293-314.

3 Wells JA, Cunningham BC, Fuh G, Lowman HB, Bass SH, Mulkerrin MG et al. The molecular basis for growth hormonereceptor interactions. Recent Progress in Hormone Research 1993 $48253-275$.

4 Abdel-Meguid SS, Shieh HS, Smith WW, Dayringer HE, Violand BN \& Bentle LA. Three-dimensional structure of a genetically engineered variant of porcine growth hormone. PNAS $1993846434-6437$.

5 Leung DW, Spencer SA, Cachianes G, Hammonds RG, Collins C, Henzel WJ et al. Growth hormone receptor and serum binding protein: purification, cloning and expression. Nature 1987330 537-543.

6 Ultsch M, De Vos AM \& Kossiakoff AA. Crystals of the complex between human growth hormone and the extracellular domain of its receptor. Journal of Molecular Biology 1991 222 865-868.

7 Cunningham BC, Ultsch M, De Vos AM, Mulkerrin MG, Clauser KR \& Wells JA. Dimerization of the extracellular domain of the human growth hormone receptor by a single hormone molecule. Science $1991254821-825$.

8 De Vos AM, Ultsch M \& Kossiakoff AA. Human growth hormone and extracellular domain of its receptor: crystal structure of the complex. Science 1992255 306-312.

9 Tou JS, Kaempfe LA, Vineyard BD, Buonomo FC, Della-Fera MA \& Baile CA. Amphiphilic growth hormone releasing factor (GRF) analogs: peptide design and biological activity in vivo. Biochemical and Biophysical Research Communications 1986139 763-770.

10 Chen WY, Wight DC, Wagner TE \& Kopchick JJ. Expression of a mutated bovine growth hormone gene suppresses growth of transgenic mice. PNAS $1990 \mathbf{8 7}$ 5061-5065.

11 Chen WY, White ME, Wagner TE \& Kopchick JJ. Functional antagonism between endogenous mouse growth hormone $(\mathrm{GH})$ and a GH analog results in dwarf transgenic mice. Endocrinology 1991 129 1402-1408.

12 Harding PA, Wang X, Okada S, Chen WY, Wan W \& Kopchick JJ. Growth hormone (GH) and a GH antagonist promote GH receptor dimerization and internalization. Journal of Biological Chemistry $19962716708-6712$.

13 Ross RJ, Leung KC, Maamra M, Bennett W, Doyle N, Waters MJ et al. Binding and functional studies with the growth hormone receptor antagonist, B2036-PEG (pegvisomant), reveal effects of pegylation and evidence that it binds to a receptor dimer. Journal of Clinical Endocrinology and Metabolism 200186 1716-1723.

14 Chen WY, Wight DC, Mehta BV, Wagner TE \& Kopchick JJ. Glycine 119 of bovine growth hormone is critical for growth-promoting activity. Molecular Endocrinology 19915 1845-1852.

15 Chen WY, Chen N, Yun J, Wagner TE \& Kopchick JJ. In vitro and in vivo studies of the antagonistic effects of human growth hormone analogs. Journal of Biological Chemistry 1994269 $15892-15897$.

16 Chen WY, Chen NY, Yun J, Wagner TE \& Kopchick JJ. In vitro and in vivo studies of antagonistic effects of human growth hormone analogs. Journal of Biological Chemistry $199426915892-15897$.

17 Fuh G, Cunningham BC, Fukunaga R, Nagata S, Goeddel DV \& Wells JA. Rational design of potent antagonists to the human growth hormone receptor. Science 1992256 1677-1680.

18 Clark R, Olson K, Fuh G, Marian M, Mortensen D, Teshima G et al. Long-acting growth hormones produced by conjugation with polyethylene glycol. Journal of Biological Chemistry 1996271 $21969-21977$.

19 Drake WM, Parkinson C, Besser GM \& Trainer PJ. Clinical use of a growth hormone receptor antagonist in the treatment of acromegaly. Trends in Endocrinology and Metabolism 200112 408-413. 
20 Cunningham BC \& Wells JA. Rational design of receptor-specific variants of human growth hormone. PNAS $1991 \mathbf{8 8}$ 3407-3411.

21 Goffin V, Bernichtein S, Carriere O, Bennett WF, Kopchick JJ \& Kelly PA. The human growth hormone antagonist B2036 does not interact with the prolactin receptor. Endocrinology 1999 $1403853-3856$.

22 Trainer PJ, Drake WM, Katznelson L, Freda PU, Herman-Bonert V, van der Lely AJ et al. Treatment of agromegaly with the growth hormone-receptor antagonist pegvisomant. New England Journal of Medicine 2000342 1171-1177.
23 Van Der Lely AJ, Hutson R, Trainer PJ, Besser GM, Barkan A, Katznelson L et al. Long-term treatment of acromegaly with pegvisomant, a growth hormone receptor antagonist. Lancet 2001 358 1754-1759.

24 Kopchick JJ, Stevens E, Parker C \& Trainer P. Growth hormone receptor antagonists. Endocrine Reviews 200223 623-646.

Received 6 December 2002

Accepted 20 December 2002 